\title{
Risk factors for rehospitalisation in COPD: role of health status, anxiety and depression
}

\author{
G. Gudmundsson*, T. Gislason*, C. Janson\#, E. Lindberg*, R. Hallin\#, C.S. Ulrikף, \\ E. Brøndum ${ }^{\star}$, M.M. Nieminen ${ }^{+}$, T. Aine ${ }^{+}$and P. Bakke ${ }^{\S}$
}

ABSTRACT: The aim of the present study was to analyse the risk of rehospitalisation in patients with chronic obstructive pulmonary disease and associated risk factors.

This prospective study included $\mathbf{4 1 6}$ patients from a university hospital in each of the five Nordic countries. Data included demographic information, spirometry, comorbidity and 12 month followup for 406 patients. The hospital anxiety and depression scale and St. George's Respiratory Questionnaire (SGRQ) were applied to all patients.

The number of patients that had a re-admission within 12 months was 246 (60.6\%). Patients that had a re-admission had lower lung function and health status. A low forced expiratory volume in one second $\left(F E V_{1}\right)$ and health status were independent predictors for re-admission. Hazard ratio ( $\mathrm{HR}$; 95\% Cl) was $0.82(0.74-0.90)$ per $10 \%$ increase of the predicted FEV1 and $1.06(1.02-1.10)$ per 4 units increase in total SGRQ score. The risk of rehospitalisation was also increased in subjects with anxiety (HR 1.76 (1.16-2.68)) and in subjects with low health status (total SGRQ score $>60$ units). When comparing the different subscales in the SGRQ, the closest relation between the risk of rehospitalisation was seen with the activity scale (HR 1.07 (1.03-1.11) per 4 unit increase).

In patients with low health status, anxiety is an important risk factor for rehospitalisation. This may be important for patient treatment and warrants further studies.

KEYWORDS: Anxiety, chronic obstructive pulmonary disease, depression, health status, rehospitalisation, risk factors

hronic obstructive pulmonary disease (COPD) is associated with intermittent exacerbations characterised by acute deterioration in the symptoms of chronic dyspnoea, cough and sputum production. Hospitalisations because of acute exacerbations are an important part of the care of patients with COPD. They are associated with further impairment of health status [1], increased mortality [2] and high costs [3]. Rehospitalisations are common and occur in up to $60 \%$ of patients within $1 \mathrm{yr}$ of the last exacerbation [4].

Multiple studies have been conducted in order to identify risk factors for COPD hospitalisations and there is also an increasing interest in modifying the risk factors in order to reduce the rate of rehospitalisation [5]. Risk factors that have been identified in previous studies are as follows: 1) low lung function [4, 6, 7]; 2 ) increasing age [7]; 3) poor quality of life $[1,8,9]$; 4) low physical function $[4,8]$; 5) history of frequent past exacerbations [1]; 6) history of previous admissions [4, 6]; 7) under prescription of long-term oxygen therapy [6]; 8) hypercapnoea; and 9) pulmonary hypertension [10]. Interventions that decrease the risk of hospitalisations in COPD patients include vaccinations for influenza [11], smoking cessation [12] and pulmonary rehabilitation [13]. A study by DAHLÉN and JANSON [14] found that anxiety and depression were related to a higher risk of relapse in patients with asthma and COPD that were admitted for emergency treatment. There is, however, limited data available regarding the level of anxiety and depression and the risk for hospital re-admission for COPD. There is also a lack of data on re-admission rates of COPD from Northern Europe and many previous studies have included data on mostly male populations.

The aim of the present study was to analyse the risk of rehospitalisation in patients with COPD and associated risk factors with special emphasis on health status, anxiety and depression.

\section{AFFILIATIONS}

*Dept of Respiratory Medicine, Allergy and Sleep, Landspitali University Hospital, Reykjavik, Iceland.

\#Dept of Medical Sciences, Respiratory Medicine and

Allergology, Uppsala University, Akademiska Sjukhuset, Uppsala, Sweden.

"Dept of Respiratory Diseases, Hvidovre Hospital, University of Copenhagen, Copenhagen, Denmark. +Dept of Respiratory Medicine, Tampere University Hospital,

Tampere, Finland.

${ }^{\S}$ Haukeland University Hospital, Bergen, Norway.

CORRESPONDENCE

G. Gudmundsson

Dept of Respiratory Medicine

Allergy and Sleep

Landspitali University Hospital

E-7 Fossvogur

IS-108 Reykjavik

Iceland

Fax: 3545436568

E-mail: ggudmund@landspitali.is

Received:

June 292004

Accepted after revision:

April 302005

SUPPORT STATEMENT

Financial support for the present study was provided by Boehringer Ingelheim in Denmark, Norway, Sweden and Finland, the Swedish Heart and Lung Association and the Swedish Heart Lung Foundation.

European Respiratory Journal Print ISSN 0903-1936 Online ISSN 1399-3003 


\section{MATERIALS AND METHODS}

This was a prospective study of patients hospitalised with acute exacerbations of obstructive airway disease in five university hospitals in the Nordic countries. The departments included were: Dept of Respiratory Medicine and Allergology, Akademiska sjukhuset, Uppsala, Sweden; Dept of Thoracic Medicine, Haukeland University Hospital, Bergen, Norway; Dept of Respiratory Medicine, Tampere University Hospital, Tampere, Finland; Dept of Respiratory Medicine, Allergy and Sleep, Vifilstadir University Hospital, Gardabaer, Iceland; and Dept of Respiratory Medicine, Hvidovre Hospital, Copenhagen, Denmark. The study was approved by the Institute Review Board and/or an ethic committee for each institution or country. Informed consent was obtained from all the patients.

Consecutive patients from each of the participating hospitals were included provided that they had been admitted with acute exacerbations of obstructive lung disease (asthma, chronic bronchitis, chronic obstructive bronchitis or emphysema) during the year 2000-2001. An acute exacerbation was defined as a change in condition in a COPD patient from baseline of such a magnitude that it needed an acute hospital admission. In the following analysis all patients with asthma were excluded. The reported data are only from those fulfilling criteria for COPD according to the Global initiative for chronic Obstructive Pulmonary Disease (GOLD) stage I or higher [15]. Codes from International classification of diseases-10 for COPD were used. All records were reviewed by the investigators to confirm the diagnosis. Only patients that were admitted for $>24 \mathrm{~h}$ were included. All information was collected by the same person in each country who had received basic training. The same person carried out the initial and follow-up information collection in each country.

The following data were collected at discharge from the respective pulmonary departments. 1) A questionnaire that included information on smoking history, type of living and family situation (alone or with others) in addition to educational level. 2) Spirometry, body weight and height. Predicted values for forced expiratory volume in one second (FEV1) and forced vital capacity were calculated based on the European Coal and Steel Union reference values [16]. 3) Health status was assessed using the disease-specific St George's Respiratory Questionnaire (SGRQ). It has three components (symptoms, activity and impact) in addition to total score [17]. Higher scores indicate a worse health status. Anxiety and depression were evaluated using the Hospital Anxiety and Depression scale (HAD) [18], which is self-reported and has been used extensively to screen psychiatric morbidity. It has high validity when used as a screening instrument for psychiatric morbidity [19-21]. It is comprised of two parts, the first with seven questions related to anxiety and the second with seven questions related to depression. A score of eight or more on either part was used as the cut-off point for diagnosing anxiety and depression, respectively [18]. 4) From the patient records information was collected on treatment at discharge, including long-term oxygen therapy. Assessment of comorbidity was based on the diagnosis used by the treating physician. Diabetes mellitus was considered to be present if using medications for diabetes. Hypertension, ischemic heart disease or atrial fibrillation were considered to be present when diagnosed by an attending physician and the patient was using appropriate medications. 5) Enrolled patients were contacted by phone $1 \mathrm{yr}$ after discharge in order to obtain information regarding number of hospitalisations. This data was confirmed by checking hospital records. In patients that had died before the follow-up, information on hospitalisations was collected through hospital records.

\section{STATISTICS}

The Chi-squared test and an unpaired t-test were used when comparing patients that had or had not been hospitalised during the study period.

The time until re-admission was analysed by the Kaplan-Meier survival analysis and Cox regression. In the Cox regression analysis the total HAD score was used together with all the other independent variables in one model. Separate estimates were then calculated for the HAD anxiety subscales, keeping all other variables except the total HAD score in the model. The estimation of the relation between the risk of rehospitalisation on the HAD depression scale was calculated in a similar fashion in a third model.

Linear regression was used to analyse the relationship between health status and psychological status. In order to detect heterogeneity between the hospitals concerning determinants for rehospitalisation the Cox regression estimates (hazard ratio (HR)) were also calculated by the hospital and then combined, using random effect meta-analysis. A p-value of $<0.05$ was considered statistically significant.

\section{RESULTS}

In total, 416 consecutive patients with COPD were enrolled in the study and re-admission data up to 1 yr was available for 406 subjects. During the study period 57 patients died. Of these, 42 had a recorded rehospitalisation due to a COPD exacerbation before dying, nine had no recorded COPD rehospitalisation and six were lost to follow-up. The number of patients that had a re-admission within 12 months was 246 $(60.6 \%)$.

Table 1 compares the characteristics of the patients included in relation with re-admission. There was no significant difference between the groups regarding anxiety and depression. Patients who had a re-admission had lower health status with higher SGRQ scores for activity (mean \pm SD; $73 \pm 18$ versus. $59 \pm 23$ ) and impact ( $50 \pm 19$ versus $41 \pm 19$; fig. 1$)$. Those with re-admission had lower lung function, were more often using long-term oxygen and had a higher number of COPD hospitalisations in the previous 12 months than patients without re-admission (table 1). Patients with rehospitalisations were more often using nebulised therapy and oral theophyllines and less often inhaled anticholinergics on discharge from hospital than subjects that did not have re-admissions (table 1). A significant relationship was found between COPD severity (fig. 2) and health status (fig. 3), as well as the time of re-admission when this was analysed by Kaplan-Meier survival analysis.

No association was found between psychological status and re-admissions when analysing data from all subjects. A significant correlation was, however, found between the total SGRQ score and the total HAD score $(r=0.38 ; \mathrm{p}<0.0001)$. Using the median of the total SGRQ score the patients were divided 
TABLE 1 Characteristics of patients included in the study in relation to re-admission

\begin{tabular}{|c|c|c|c|c|}
\hline Subjects $n$ & 406 & 160 & 246 & \\
\hline Female & 51.2 & 54.4 & 49.2 & 0.31 \\
\hline Hospital stay days & $8(6-13)$ & $7(5-12)$ & $9(6-14)$ & 0.02 \\
\hline Smokers & 36.0 & 30.6 & 23.0 & 0.09 \\
\hline Living alone & 52.1 & 48.7 & 54.4 & 0.28 \\
\hline COPD hospitalisations in last 12 months & $1(0-3)$ & $0.5(0-1)$ & $1(1-3)$ & $<0.0001$ \\
\hline HAD-Total & $12.5 \pm 6.8$ & $12.1 \pm 6.3$ & $12.7 \pm 7.1$ & 0.38 \\
\hline HAD-Anxiety & $7.0 \pm 4.2$ & $6.7 \pm 4.0$ & $7.1 \pm 4.3$ & 0.28 \\
\hline HAD-Depression & $5.5 \pm 3.6$ & $5.4 \pm 3.4$ & $5.6 \pm 3.8$ & 0.63 \\
\hline FVC $\%$ of pred & $61.8 \pm 19.2$ & $66.2 \pm 19.2$ & $58.8 \pm 20.7$ & 0.0005 \\
\hline Long-term oxygen & 23.6 & 16.2 & 28.4 & 0.005 \\
\hline Cardiovascular diseases & 44.6 & 45.6 & 43.9 & 0.76 \\
\hline Diabetes & 10.3 & 7.5 & 12.2 & 0.13 \\
\hline Anticholinergics & 35.8 & 43.6 & 30.7 & 0.009 \\
\hline Short acting $\beta_{2}$-agonists & 35.9 & 33.8 & 37.3 & 0.53 \\
\hline Long acting $\beta_{2}$-agonists & 61.4 & 60.5 & 61.9 & 0.78 \\
\hline Inhaled corticosteroids & 69.9 & 69.9 & 70.0 & 0.98 \\
\hline Nebulised treatment & 33.2 & 19.9 & 41.8 & $<0.0001$ \\
\hline Oral theophylline & 27.7 & 19.7 & 32.9 & 0.004 \\
\hline Oral corticosteroids & 65.7 & 62.4 & 67.9 & 0.26 \\
\hline
\end{tabular}

Data presented as $n$, mean \pm SD, median (interquartile range) or \%. COPD: chronic obstructive pulmonary disease; HAD: hospital anxiety and depression scale; SGRQ: St George's Respiratory Questionnaire; FEV1: forced expiratory volume in one second; FVC: forced vital capacity. Chi-squared test and unpaired t-tests was used to calculate the $p$-values.

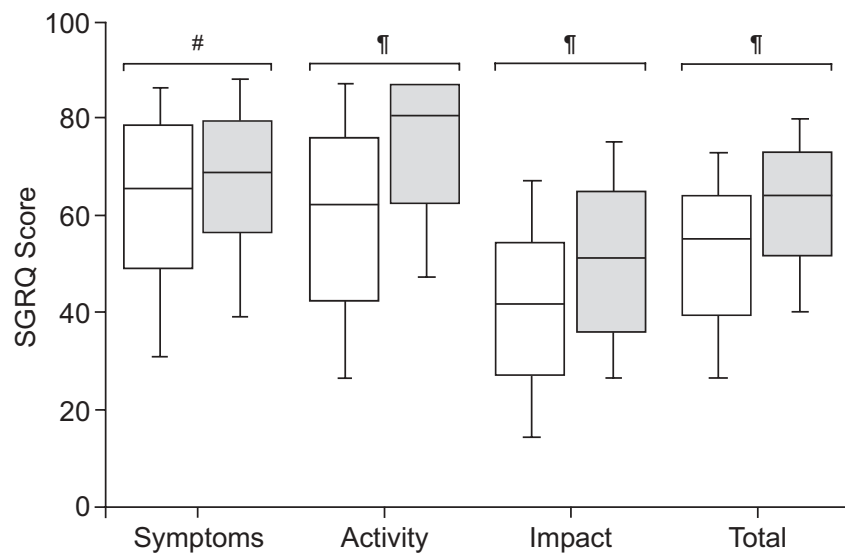

FIGURE 1. Health status in relation to patients with $(\square)$ and without $(\square)$ hospital re-admissions. The box plot displays the median, 10, 25, 50, 75 and $90 \%$ percentiles. ${ }^{*}: p=0.08 ;{ }^{\natural}: p<0.0001$.

into patients with higher (total SGRQ score $\leqslant 60$ units) and lower (total SGRQ $>60$ units) health status. There was a significant interaction between health status and psychological status in relation to the risk of re-admission $(\mathrm{p}=0.002)$ with a significant correlation between the total HAD score and the

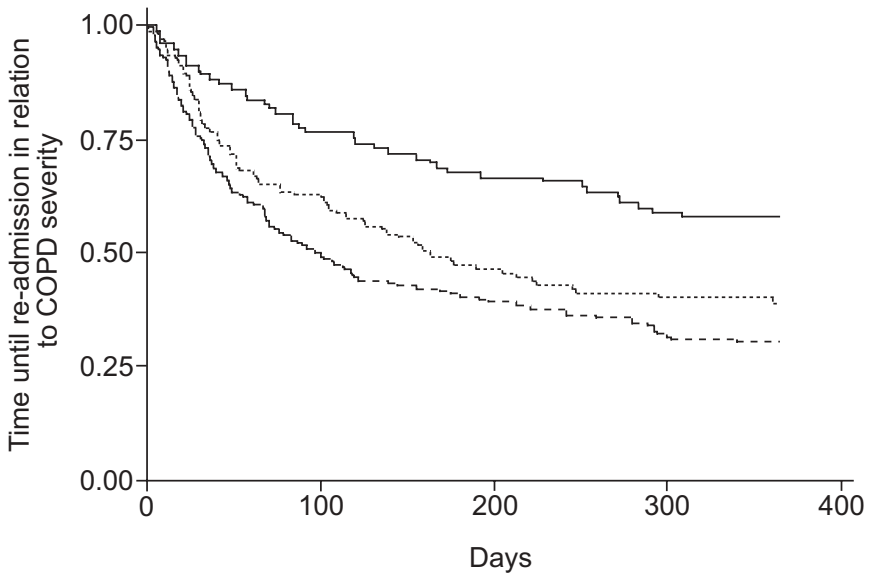

FIGURE 2. Kaplan-Meier survival curve demonstrating the time until readmission in patients in relation to chronic obstructive pulmonary disease (COPD) severity defined according to the Global initiative for chronic Obstructive Lung Disease (GOLD) classification. —-: GOLD I and II; . . : GOLD III; - - -: GOLD IV. p<0.0001.

risk of re-admission in patients with a low health status (table 2). In the groups of subjects with low health status, higher anxiety score was related to increased risk of 


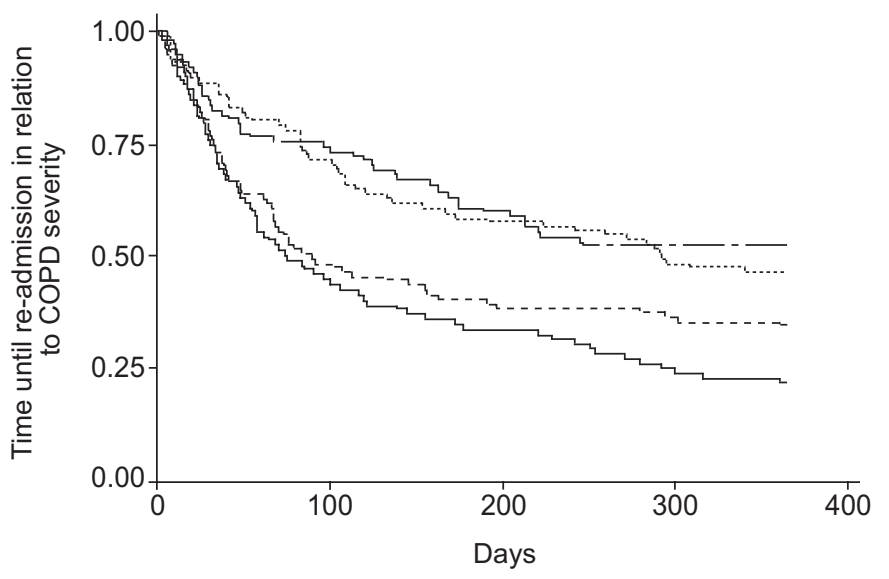

FIGURE 3. Kaplan-Meier survival curve demonstrating time until re-admission in relation to health status (St George's Respiratory Questionnaire (SGRQ) total score). The patients were divided into quartiles according the SGRQ total score. - - -: 1st quartile $<46.4 ; \cdots$. 2nd quartile 46.4-60.4; - - -: 3rd quartile 60.5-70.5; —: :th quartile $>70.6 . p<0.0001$

\begin{tabular}{|c|c|c|c|}
\hline \multirow[t]{2}{*}{ TABLE 2} & \multirow[b]{2}{*}{ All subjects } & \multirow[b]{2}{*}{$\begin{array}{l}\text { SGRQ total } \\
\quad \leqslant 60\end{array}$} & \multirow[b]{2}{*}{$\begin{array}{l}\text { SGRQ total } \\
>60\end{array}$} \\
\hline & & & \\
\hline Subjects n & 340 & 170 & 170 \\
\hline Age $10 \mathrm{yrs}$ & $1.16(1.00-1.35)$ & $1.14(0.92-1.419)$ & $1.19(0.95-1.50)$ \\
\hline Current smoker & $0.78(0.55-1.10)$ & $0.69(0.37-1.29)$ & $0.82(0.53-1.26)$ \\
\hline HAD-total 4 units* & $1.03(0.93-1.13)$ & $0.87(0.74-1.03)$ & $1.13(1.01-1.27)$ \\
\hline HAD-anxiety 4 units* & $1.10(0.95-1.28)$ & $0.81(0.63-1.04)$ & $1.36(1.12-1.65)$ \\
\hline $\begin{array}{l}\text { HAD-depression } \\
4 \text { units* }\end{array}$ & $0.96(0.80-1.15)$ & $0.83(0.61-1.14)$ & $1.02(0.81-1.28)$ \\
\hline Anxiety* & $1.06(0.79-1.41)$ & $0.43(0.25-0.74)$ & $1.73(1.18-2.53)$ \\
\hline Depression* & $1.09(0.80-1.51)$ & $0.84(0.46-1.55)$ & $1.20(0.81-1.79)$ \\
\hline FEV $110 \%$ pred & $0.83(0.76-0.91)$ & $0.80(0.70-0.92)$ & $0.83(0.53-1.26)$ \\
\hline SGRQ-total 4 units & $1.06(1.02-1.11)$ & $1.06(0.97-1.15)$ & $1.08(0.98-1.18)$ \\
\hline
\end{tabular}

Data presented as $\mathrm{n}$ and hazard ratio (95\% confidence interval). SGRQ: St George's Respiratory Questionnaire; HAD: hospital anxiety and depression scale; FEV1: forced expiratory volume in one second. *: Entered separately into the model.

rehospitalisation. No significant relationship between depression score and the risk of rehospitalisation was found regardless of health status (table 2).

FEV1 and health status were independent predictors for readmission in the multivariable analysis (table 2). Previous hospitalisations were also independently related to an increased risk of rehospitalisation for at least two previous hospitalisations (HR (95\% confidence interval (CI)): 1.98 (1.422.76)). Including previous hospitalisations, however, did not change the relationship between FEV1, health status and the risk of rehospitalisations.

There was no change in the associations between FEV1, health status, psychological status and the risk of rehospitalisation after adding in the pharmacological treatment.
None of the different types of treatment were significantly associated with re-admission after adjusting for the other covariables.

When comparing the different subscales in the SGRQ, no association was found between the symptom scale and readmission (HR (95\% CI) for a 4 unit increase 1.01 (0.98-1.05)), while a significantly increased risk for re-admissions was found both for the impact (1.04 (1.01-1.07)) and the activity scale (1.07 (1.03-1.11)). There was no significant difference between the hospitals in the association between risk of readmission and FEV1 or health status ( $p$-value for heterogeneity $=0.30$ and 0.68 , respectively).

\section{DISCUSSION}

The main finding of the present prospective multicentre study was that in COPD patients with low health status being discharged after hospital admission, the risk of rehospitalisation was higher in patients with anxiety. A low FEV1 and low health status were found to be important risk factors for readmission.

It is unclear if the prevalence of depression is increased in COPD patients in general, but the prevalence of anxiety is increased in COPD patients compared with control groups $[22,23]$. The symptoms of COPD such as breathlessness, limitation of activity and repeated exacerbations are likely to cause anxiety in patients with COPD. Cigarette smoking is also increased in patients with anxiety, thus, psychiatric disturbance is an aetiologic agent in COPD [24]. Hospitalised patients with COPD have a high prevalence of depression and anxiety [25]. The association of depression and anxiety with risk of rehospitalisation is an area that has not been well studied previously. In the present study there was a significant association between anxiety, depression and health status. In patients who had a low health status a significant increased risk of rehospitalisations was found in those patients that had anxiety. It is possible that the low health status causes anxiety in these patients or that they were anxious previously. No association was found between depression and the risk of re-admissions. In contrast to the current results no association was found with risk of readmission using the mental scale of short form (SF)-12 or the mental health inventory of the SF-36 in the Estudi del Factors de Risc d'Aguditzacio de la MPOC (EFRAM) study [4]. FAN et al. [9] found that patients with the lowest scores on the emotional function skills scale in health status were at greater risk for rehospitalisation. A study by OKUBADEJO et al. [26] found that in patients with COPD and severe hypoxemia the SGRQ total score was related with anxiety and depression measured by the HAD scale. Multivariate analysis showed that depression score and arterial oxygen tension were both significant covariates of the SGRQ total score. There is lack of standardisation for instruments to detect anxiety and depression and the definition of anxiety and depression. The HAD scale was used in the present study with a previously recognised cut-off score for significant disease [18]. The current authors have used the HAD scale previously in a study on a mixed population of asthmatics and COPD patients where anxiety and depression were found to increase the risk of relapse after emergency treatment [14]. 
The present study shows that health status is an important risk factor for rehospitalisation. This is consistent with previous studies $[1,8,9]$. OSMAN et al. [8] found that higher scores on the SGRQ (lower health status) predicted hospital re-admission within the next 12 months, while sex, age, and pulmonary function did not.

It is of interest that the association of health status was highest with the activity score on the SGRQ. The EFRAM study from Spain showed that higher levels of physical activity reduced the risk for rehospitalisation [4]. Patients that reported an activity equivalent to walking for $\geqslant 1 \mathrm{~h}$ a day had a reduction in risk of admission to hospital of almost $50 \%$. The same was true for those who scored highly on the physical scale of SF-36 [4]. In another study FAN et al. [9] showed that patients with lowest physical function had an odds ratio of 6.0 for hospitalisation. It is, therefore, likely that the high score on activity in the current study, suggesting severe limitation in activity, shows that deconditioning is a risk factor for COPD admission.

The results that hospitalised patients with a low FEV1 have a higher risk of being readmitted is in accordance with several previous studies that have shown that impaired lung function is an important risk factor for hospitalisations [4, 6, 7, 27]. As in previous studies patients with frequent previous hospitalisations were found to have a higher risk of being rehospitalised $[4,6,27]$.

ROBERTS et al. [27] found that patients with a higher number of medications and patients using home nebulisers had a higher risk of re-admission [27]. This probably reflects severity of disease, with these medications reserved for the patients with the most severe disease rather than the effect of the medications. No independent relation was found between pharmacological treatment and the risk of re-admissions after adjusting other covariables such as FEV1, health status and psychological status. Neither was any relationship found between socioeconomic variables such as educational level or home situation and the risk of rehospitalisations.

The re-admission rate of $60 \%$ is very similar to other studies. A retrospective study from Hong Kong on 551 patients showed a $59.35 \%$ re-admission rate after 12 months [28]. In the prospective EFRAM study, $63 \%$ of 340 patients were readmitted during the 1yr follow-up time [4].

The strengths of the present study are the large number of patients included, making it the largest prospective survey on COPD re-admission ever. Only 10 out of 416 (2\%) patients were lost to follow-up. The study included patients from five countries, a mixture of males and females and different GOLD stages. Similar relationships were observed between the risk factors and COPD re-admission in all the countries. There were several limitations to the study, including lack of information on participants versus nonparticipants, and cointervention such as nonpharmacological treatments e.g. rehabilitation and education. The HAD questionnaire is fairly short and naturally has some limitation in diagnosing anxiety and depression. The instrument has, however, in previous studies been shown to have a fairly high validity when used as a screening instrument for psychiatric morbidity [19-21].
In conclusion, psychological status is important in patients with chronic obstructive pulmonary disease. There is a clear association between health status and anxiety and depression, and an increased risk of rehospitalisations in patients with anxiety who have a low health status. This adds important new information in the treatment of hospitalised patients with chronic obstructive pulmonary disease and on the risk factors of rehospitalisation. Further studies are necessary to observe if treatment of anxiety can reduce the rate of rehospitalisation in patients with chronic obstructive pulmonary disease. Lung function and health status are the most important risk factors for re-admission and the present study underlines the high readmission rates for patients admitted for chronic obstructive pulmonary disease exacerbation within $1 \mathrm{yr}$.

\section{ACKNOWLEDGEMENTS}

The authors wish to thank all the participants in the study.

\section{REFERENCES}

1 Seemungal TAR, Donaldson GC, Paul EA, Bestall JC, Jeffries DJ, Wedzicha JA. Effects of exacerbation on quality of life in patients with chronic obstructive pulmonary disease. Am J Respir Crit Care Med 1998; 157: 1418-1422.

2 Connors AF, Dawson NV, Thomas C, et al. Outcomes following acute exacerbation of severe chronic obstructive disease. Am J Respir Crit Care Med 1996; 154: 959-967.

3 Anderson F, Borg S, Jansson SA, et al. The costs of exacerbations in chronic obstructive pulmonary disease. Resp Med 2002; 96: 700-708.

4 Garcia-Aymerich J, Farrero E, Felez MA. Risk factors of readmission to hospital for a COPD exacerbation: a prospective study. Thorax 2003; 58: 100-105.

5 Garcia-Aymerich J, Barreiro E, Farrero E. Patients hospitalized for COPD have a high prevalence of modifiable risk factors for exacerbation. Eur Respir J 2000; 16: 1037-1042.

6 Garcia-Aymerich J, Monsó E, Marrades RM, et al. Risk factors for hospitalisation for a chronic obstructive pulmonary disease exacerbation. Am J Respir Crit Care Med 2001; 164: 1002-1007.

7 Miravitlles M, Guerrero T, Mayordomo C, SánchezAgudo L, Nicolau F, Segú JL. Factors associated with increased risk of exacerbation and hospital admission in a cohort of ambulatory COPD patients: a multiple logistic regression analysis. Respiration 2000; 67: 495-501.

8 Osman LM, Godden DJ, Friend JAR, Legge JS, Douglas JG. Quality of life and hospital re-admission in patients with chronic obstructive pulmonary disease. Thorax 1997; 52: 67-71.

9 Fan VS, Curtis JR, Tu SP, McDonell MB, Fihn SD. Using quality of life to predict hospitalisation and mortality in patients with obstructive lung diseases. Chest 2002; 122: 429-436.

10 Kessler R, Faller M, Fourgaut G, Mennecier B, Weitzenblum E. Predictive factors of hospitalization for acute exacerbation in a series of 64 patients with chronic obstructive pulmonary disease. Am J Respir Crit Care Med 1999; 159: 158-164.

11 Nichol KL, Baken L, Nelson A. Relation between influenza vaccination and outpatient visits, hospitalisation, and 
mortality in elderly persons with chronic lung disease. Ann Intern Med 1999; 130: 397-403.

12 Godtfredsen NS, Vestbo J, Osler M, Prescott E. Risk of hospital admission for COPD following smoking cessation and reduction: a Danish population study. Thorax 2002; 57: 967-972.

13 Lacasse Y, Wong E, Guyatt GH, King D, Cook DJ, Goldstein RS. Metaanalysis on respiratory rehabilitation in chronic obstructive pulmonary disease. Lancet 1996; 348: 1115-1119.

14 Dahlén I, Janson C. Anxiety and depression are related to the outcome of emergency treatment in patients with obstructive pulmonary disease. Chest 2002; 122: 1633-1637.

15 Pauwels RA, Buist AS, Calverley PM, Jenkins CR, Hurd SS. Global strategy for the diagnosis, management and prevention of chronic obstructive pulmonary disease. NHLBI/WHO Global Initiative for Chronic Obstructive Lung Disease (GOLD) workshop summary. Am J Respir Crit Care Med 2001; 163: 1256-1276.

16 European Community for Coal and Steel. Standardisation of lung function tests. Clin Respir Phys 1983; 19: Suppl. 5, 22-27.

17 Jones PW, Quirk FH, Baveystock CM, Littlejohns P. A selfcomplete measure of health status for chronic airflow limitation. The St. Georges Respiratory Questionnaire. Am Rev Respir Dis 1992; 145: 1321-1327.

18 Zigmond AS, Snaith RP. The hospital anxiety and depression scale. Acta Psyciatr Scand 1983; 67: 361-370.

19 Wilkinson MJ, Barczak P. Psychiatric screening in general practice: comparison of the general health questionnaire and the hospital anxiety depression scale. J R Coll Gen Pract 1988; 38: 311-313.
20 Moorey S, Greer S, Watson M, et al. The factor structure and factor stability of the hospital anxiety and depression scale in patients with cancer. Br J Psychiatry 1991; 158: 255-259.

21 Dowson C, Laing R, Barraclough R, et al. The use of the Hospital Anxiety and Depression Scale (HADS) in patients with chronic obstructive pulmonary disease: a pilot study. N Zealand Med J 2001; 114: 447-449.

22 van Ede L, Yzermans CJ, Brouwer HJ. Prevalence of depression in patients with chronic obstructive pulmonary disease: a systematic review. Thorax 1999; 54: 688-692.

23 Brenes GA. Anxiety and chronic obstructive pulmonary disease: prevalence, impact and treatment. Psychosom Med 2003; 65: 963-970.

24 Lasser K, Boyd JW, Woolhandler S, et al. Smoking and mental illness. A population-based prevalence study. JAMA 2000; 284: 2606-2610.

25 Aghanwa HS, Erhabor GE. Specific psychiatric morbidity among patients with chronic obstructive pulmonary disease in a Nigerian general hospital. J Psychosom Res 2001; 50: 179-183.

26 Okubadejo AA, Jones PW, Wedzicha JA. Quality of life in patients with chronic obstructive lung disease and severe hypoxaemia. Thorax 1996; 51: 44-47.

27 Roberts CM, Lowe D, Bucknall CE, Ryland I, Kelly Y, Pearson MG. Clinical audit indicators of outcome following admission to hospital with acute exacerbation of chronic obstructive pulmonary disease. Thorax 2002; 57: 137-141.

28 Lau AC-W, Yam Y-C, Poon E. Hospital re-admission in patients with acute exacerbation of chronic obstructive pulmonary disease. Resp Med 2001; 95: 876-884. 